\title{
An unexpected finding in a diabetic patient studied with transthoracic echocardiography
}

\begin{abstract}
Coronary artery fistulas comprise can be can be either congenital or acquired. Congenital fistulas represent about $14 \%$ of congenital coronary artery anomalies, but they are seen also in approximately 0.1 to $0.2 \%$ of all patients undergoing selective coronary angiography. Then, acquired fistulas have been reported as a complication of deceleration accidents, percutaneous transluminal coronary angioplasty or cardiac surgery. We describe a case we evaluated through transthoracic echocardiographic technique (ultrasound findings confirmed by transesophageal technique).
\end{abstract}

Keywords: coronary artery fistula, congenital heart disease, echocardiography, coronary atherosclerosis, catheterization
Volume 2 Issue I - 2017

\author{
Valerio Massimo Magro,' Fulvio Cacciapuoti, ${ }^{2}$ \\ Federico Cacciapuoti,' Diana Lama' \\ 'Department of Internal Medicine and Geriatry, Second \\ University of Naples, Italy \\ ${ }^{2}$ Azienda Ospedaliera di Rilievo Nazionale Antonio Cardarelli, \\ Italy
}

Correspondence: Valerio Massimo Magro, Department of Internal Medicine and Geriatry, Second University of Naples, Italy, Tel 3492224922, Email valerio_magro@hotmail.com

Received: May 20, 2017 | Published: August 23, 2017

\section{Case report}

An 74-year-old man (former smocker for about 27years) was referred for evaluation of an exertional dyspnoea. He reported recent history of few episodes of shortness of breath associated with moderate entity physical activity. In medical history presence of type 2 diabetes with poor glycemic control ( $\mathrm{HbA} 1 \mathrm{c} 7.7 \%$ ) and micro- and macrovascular complications: carotid stenosis in the internal carotid level bilaterally with obstruction of $40 \%$ and $17 \%$, on the left and on the right, respectively, femoral and popliteal atherosclerosis. The subject had gone several times to meet catheterization procedures such as catheter ablation of atrial fibrillation four years before and a previous (six years before) acute myocardial infarction (AMI) treated with percutaneous coronary intervention (PCI). At physical examination the patient appeared in good clinical conditions. We could hear a 2/6 murmur on the auscultation mitral outbreak. A transthoracic echocardiogram showed a normal size left ventricle with a small hypokinetic area at the level of the lower wall; Eiection Fraction (EF) $>50 \%$ (the systolic function of the left ventricle was preserved). Hypertrophy of the interventricular septum and left atrial enlargement (Vol LA/BSA $28 \mathrm{ml} / \mathrm{mq}$ )(Figure 1). Aortic, Mitral, tricuspid and pulmonary regurgitation. We identified the outlet of the right coronary artery, with well viewed blood flow (Figure 2). The exam also showed (Figures 3-4) a flow at color Doppler analysis in the high parasternal short axis view, originating from a small entry site on the wall of the main pulmonary artery. We suspected the presence of a fistula connecting the portion of the aortic root, at the level of the left coronary artery outlet, with the rear portion of the pulmonary artery or alternatively the presence of a vascular coronary wall damage. At the beginning we were not convinced and we thought it might be a pulmonary valve insufficiency with double jet but the absence of apparent partial discrepancy seen with the operator's eye between the jets in the same phase of the cardiac cycle forced us to continue our evaluation anyway, so that we decided to initiate the patient to a subsequent study with other imaging (transesophageal echocardiography; myocardial computed tomography). The next transesophageal echocardiography put well in evidence a diastolic flow in the proximal tract of the pulmonary trunk, confirming the suspicion of the coronary fistula, which was judged not hemodynamically significant. So we decided to prefer a cautious annual follow-up.
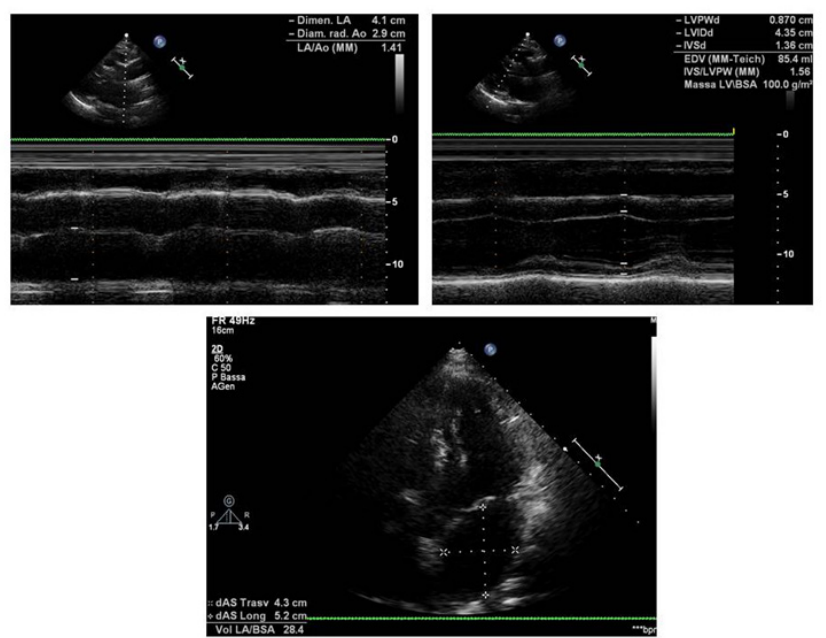

Figure I Hypertrophy of the interventricular septum and left atrial enlargement.

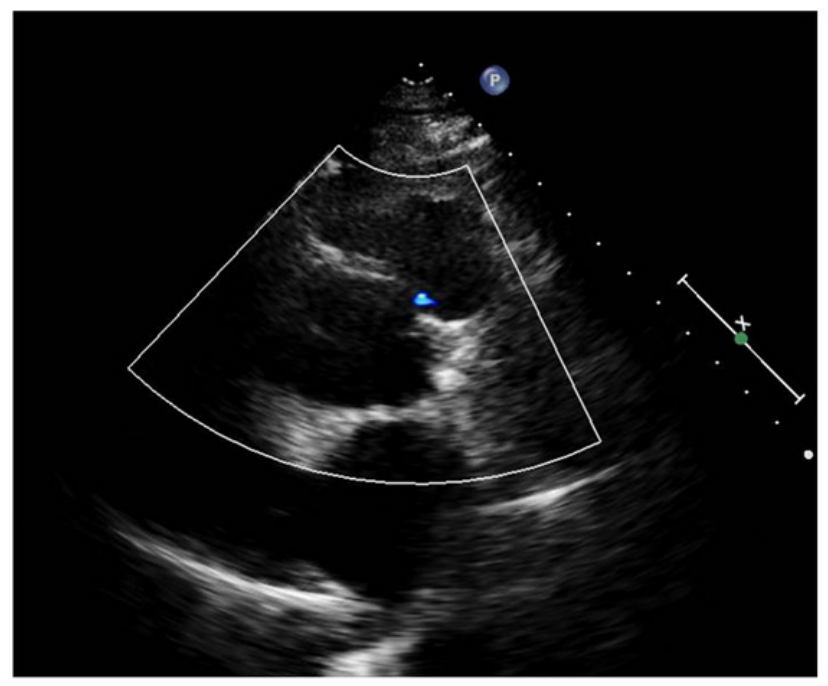

Figure 2 Outlet of the right coronary artery, with well viewed blood flow. 

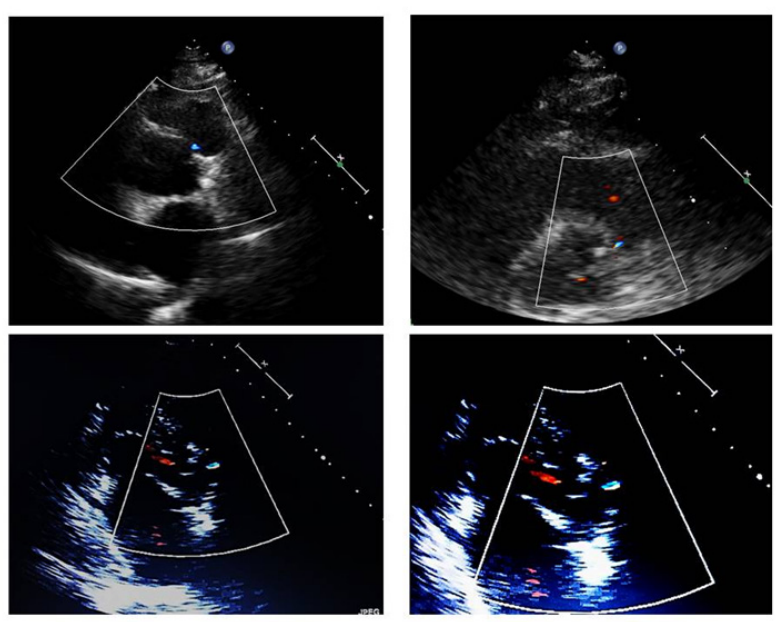

Figure 3 Color Doppler analysis in the high para sternal short axis view.
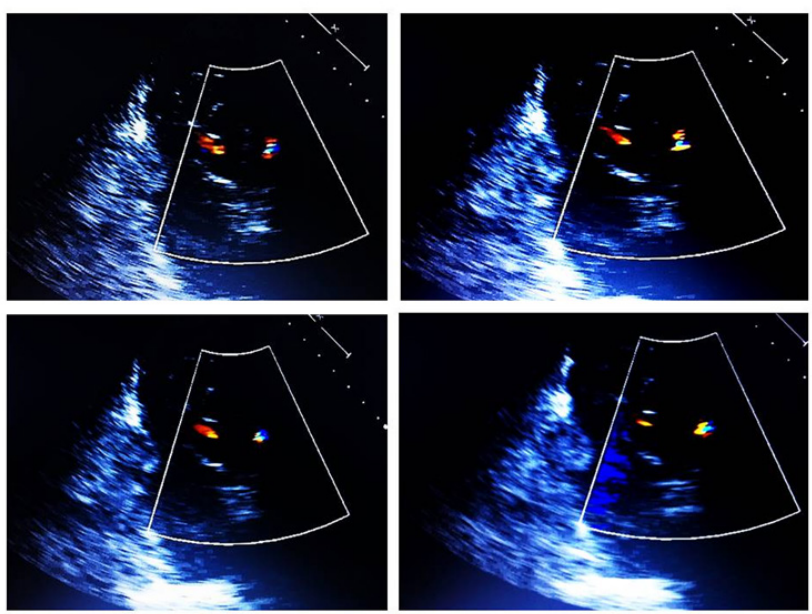

Figure 4 Originating from a small entry site on the wall of the main pulmonary artery.

\section{Discussion}

\section{Clinical features}

Coronary artery fistulas are unusual congenital or acquired coronary artery abnormalities in which blood is shunted into a cardiac chamber or great vessel. They are usually congenital, but acquired forms do occur especially in the patient to adulthood. ${ }^{1,2}$ Their clinical importance usually in the adulthood is due to an increased risk of complications, including heart failure, myocardial ischemia, infective endocarditis, arrhythmias, and rupture. The acquired causes of coronary fistulas include coronary atherosclerosis (our patient, with diabetes, was marked by a multi district atherosclerotic disease and also coronary atherosclerotic damage, and his history showed the patient was already suffering from AMI), Takayasu arteritis, and trauma. ${ }^{3,4}$ About trauma we must also remember that the patient had been subjected in several times to long procedures that used catheterization (PCI, ablation). The formation of fistulas is reported as a documented complication of PCI, although most frequently to access level (femoral vessel). ${ }^{5}$ In the examined case report, it can also be a suspicion of iatrogenic origin of the observed anomaly. Trauma of coronary artery wall due to the fact invasive cardiology procedures can in fact determine the formation of fistulas or the delamination of the robes of the coronary vessel, causing a coronary artery dissection, or both. The blood microinfiltration from endoluminal space to intima can cause a dissecation of the vessel and thus breaking.

\section{Radiological and instrumental study}

We describe a case we evaluated through transthoracic echocardiographic technique. However, several limitations have been described with transthoracic echocardiography in patients with coronary artery fistulas. Color Doppler may not detect flow in the distal site of coronary artery fistulas. Multiplane transesophageal echocardiography can more accurately define and provide a high quality panoramic view of the origin, course, and drainage site of coronary artery fistulas. New imaging modalities, using contrast enhanced ultrafast computed tomography or magnetic resonance imaging can be used as an addition or replacement to coronary angiography. ${ }^{6-8}$ However, it is necessary a multi-imaging approach to achieve accurate diagnosis and to definite the anatomical relationships so that we can plan the best therapeutical strategy. On the other hand, treatment of adult asymptomatic patients with non-significant shunting is still a matter of debate. Catheter-based closure of the fistulous connection is the non-surgical treatment option for closure of coronary fistulas, with good reported success. ${ }^{9-11}$

\section{Conclusion}

Coronary artery fistula has been described as a direct connection between a coronary artery and one of the cardiac chambers, large vessels or other vascular structures. The coronary artery fistula between single left coronary artery and the pulmonary artery has not been frequently reported and it is a rare anomaly. However it can be congenital but also acquired. We presented a case report who came to our attention and studied by echocardiography. We believe that coronary artery fistula is an infrequent but significant pathology which needs to be investigated. Its diagnosis is important because of the potential therapeutic implications. As with many other coronary anomalies, together with the angiography (gold standard), echocardiography techniques (that are the first level of diagnostic suspicion as this case report exemplifies) and coronary or aortic root computed tomography are the main methods for the diagnosis.

\section{Acknowledgements}

None.

\section{Conflict of interest}

Authors declare there is no conflict of interest in composing this manuscript.

\section{References}

1. Gowda RM, Vasavada BC, Khan IA. Coronary artery fistulas: clinical and therapeutic considerations. Int J Cardiol. 2006;107(1):7-10.

2. Dehaene A, Jacquier A, Falque C, et al. Imaging of acquired coronary diseases: from children to adults. Diagn Interv Imaging. 2016;97(5):571-580.

3. Said SA, Van der Werf T. Acquired coronary cameral fistulas: are these collaterals losing their destination. Clin Cardiol. 1999;22:297-302.

4. Said SA, Gamal MI, Werf T. Coronary arteriovenous fistulas: collective review and management of six new cases-Changing etiology, presentation, and tratment strategy. Clin Cardiol. 1997;20(9):748-752. 
5. Iannone LA, Iannone DP. Iatrogenic left coronary artery fistula-to-left ventricle following PTCA: a previously unreported complication with non surgical management. Am Heart J. 1990;5:1215-1217.

6. Lim WH, Kang SH, Jeon K, et al. Asymptomatic right coronary arteryto-pulmonary artery fistula incidentally detected by transthoracic echocardiography. J Cardiovasc Ultrasound. 17(3):106-109.

7. Chen BH, Lin CC, Weng KP, et al. Echocardiographic diagnosis of incidentally found left coronary artery to pulmonary artery fistula in an 11.year-old girl. Acta Cardiol Sin. 2016;32(3):359-362.

8. Vitarelli A, De Curtis G, Conde Y, et al. Assessment of congenital coronary artery fistulas by transesophageal color Doppler echocardiography. Am J Med. 2002;113(2):127-133.
9. Jiritano F, Prestipino F, Mastroroberto P, et al. Coronary arterovenous fistula: to treat or not to treat? J Cardiothorac Surg. 2015;10:52.

10. Carrel T, Tkebuchava T, Jenni R, et al. Congenital coronary fistulas in children and adults: diagnosis, surgical technique and results. Cardiology. 1996;87(4):325-330.

11. Kulkarni SM, Parmar RC. Catheter interventions in miscellaneous lesions in CHD: ruptured sinus of Valsalva, coronary arteriovenous fistulas, and pulmonary arteriovenous fistulas. Indian Heart J. 2008;60(4 Suppl D):34-446. 